\title{
Differentiation and diagnosis of migrating cerebral sparganosis: 2 case reports from China
}

\author{
Xunya Hou ${ }^{1}$, Donald P. McManus ${ }^{2}$, Zhaoyang Lou ${ }^{1}$, Sixi Chen ${ }^{1}$, Yuesheng Li ${ }^{1,2 \#}$ \\ ${ }^{1}$ Hunan Institute of Parasitic Diseases, National Key Center on Endemic Diseases in Xiangyue Hospital, Hunan Key Laboratory of \\ Immunology and Transmission Control on Schistosomiasis, Yueyang, China; \\ \#Corresponding Author: yuesheng_li@yahoo.com.au \\ ${ }^{2}$ Molecular Parasitology Laboratory, Infectious Diseases Division, Queensland Institute of Medical Research, Brisbane, Australia
}

Received 12 October 2012; revised 15 November 2012; accepted 25 November 2012

\begin{abstract}
Human cerebral sparganosis is a rare but neglected parasitic disease. We present details of the diagnosis of two cases of migrating cerebral sparganosis from China based on computed tomography and magnetic resonance imaging combined with epidemiological information and serology. Its differentiatial diagnosis from other parasitic diseases, tuberculoma and brain tumor by neuroimaging is briefly discribed.
\end{abstract}

Keywords: Chinese; Spirometra; Sparganosis; Cerebral Sparganosis; Diagnosis; Computed Tomography; Magnetic Resonance Imaging; Differentiation

\section{INTRODUCTION}

Human sparganosis is caused by the plerocercoid larvae (spargana) of several species of pseudophyllid cestodes belonging to the genera Spirometra [1]. Many cases of human infection are probably due to Spirometra mansoni. China is one of the most endemic counties for sparganosis in Asia. To date, a total of more than 1000 cases of human sparganosis have been reported in 25 provinces of mainland China [2,3].

The life cycle of Spirometra involves 3 hosts. The definitive hosts are carnivores such as cats and dogs. The first intermediate host is a copepod (Cyclops spp.) and the second intermediate hosts are generally frogs and snakes. Humans can act as the second intermediate or paratenic host and become infected with spargana by drinking water contaminated with procercoid-infected copepods, by ingesting undercooked frog or snake infected with spargana, or by local application of frog or snake flesh or skin as poultices to open wounds and other lesions or (especially) to sore eyes; the larvae migrate

*Competing interests: the authors declare that they have no competing interests. into the human tissue from the poultice. Spirometra can infect various parts of the human body such as the eyes, buccal and maxillofacial subcutaneous tissues, visceral organs and the central nervous system (CNS). Cerebral sparganosis, which occurs when spargana invade the brain, can result in severe tissue damage and even death. The predominant manifestations are fever, headache and vomiting, as well as neck stiffness and Kernig's sign. Seizures are common in $16 \%-87.8 \%$ of patients with cerebral sparganosis. Other symptoms include visual disturbances, motor weakness and sensory disturbance [1-3]. Here we describe the diagnosis of two cases of migrating cerebral sparganosis based on the characteristics of computed tomography (CT) and brain magnetic resonance imaging (MRI), complemented by epidemiological information and serology.

\section{CASE REPORT}

\subsection{Case}

In May 2011, a 13-year-old male from Jiangxi province presented to the affiliated Xiangyu Hospital of Hunan Institute of Parasitic Diseases (HIPD), Yueyang, Hunan province complaining of having discontinuous headaches for 6 years and having seizures on 6 occasions since 2005. Each seizure lasted about 5 - 6 minutes with loss of consciousness and strabismus but no gatism and no foaming from the mouth. In 2007, he had aphasia for a few minutes. He was tentatively diagnosed as having one of the cerebral parasitic diseases and was treated locally with oral praziquantel (total dose: $60 \mathrm{mg} / \mathrm{kg}$ ) twice in 2005 and 2006. Other treatment included oral albendazole (one dose of $400 \mathrm{mg}$ ) and the anti-convulsant sodium valproate ( $0.1 \mathrm{~g}$ dose, three times a day for 4 years until hospital admission in 2011).

His medical history and the results of physical examination, laboratory tests, including serology and skin tuberculin test for tuberculosis (TB) (PPD test), indicated that the patient had no hepatitis B or C (HBV and HCV) infection, TB or any other infectious disease including 
HIV, gonorrhoea or syphilis. Antibody serology, by enzyme-linked immunosorbent assay (ELISA) of serum and cerebrospinal fluid (CSF), for 5 parasitic diseases (schistosomiasis, echinococcosis, taeniasis solium, taeniasis bovis, toxoplasmosis) was negative but positive to soluble antigen of Spirometra mansoni.

Information obtained from the patient's mother revealed that he often attended large gatherings where frogs were eaten and may not have been cooked adequately. She further indicated that he never drank unboiled water nor did he have any history of applying frog or snake flesh to any wound.

An initial Axial MRI at the onset of disease symptoms (October, 2005) showed a low signal lesion on T1weighted spin-echo images (T1WI) (Figure 1(A)) and a heterogeneous high signal on T2WI (Figure 1(B)) in the left temporo-occipital lobe. Edema and widening of local sulci were revealed on both T1WI and T2WI (Figures 1(A) and (B)). Initial sagittal enhanced MRI (Figure 1(C)) showed a string of bead-shape or ring-like lesions (tunnel sign) in the left temporo-occipital lobe and abnormal enhancement of the adjacent dura mater. After the onset of symptoms, serial MRI revealed that the lesions had shifted from the frontal lobe at 16-months follow-up (Figure 1(D)) and subsequently to the gyrus frontalis superior 69-months later (Figure 1(E)). An encephalo-malacia focus with signal intensity similar to that of CSF was persistent continually at the left occipital pole from initial illness to the last follow up (Figures 1(A)-(E)). Another new encephalomalacia focus was found at 69-months later in the gyrus frontalis medius and inferior (Figure 1(E)). Unenhanced CT undertaken in May 2011 (Figure 1(F)) revealed low-density areas in both the left occipital and temporal lobes, and scattered pinpoint calcifications in the left cerebral hemisphere.

\subsection{Case}

A 17-year-old male from Hunan province reported to Xiangyu Hospital of HIPD having experienced seizures twice in the evening of the $3^{\text {rd }}$ and in the early morning of the $4^{\text {th }}$ October, 2010. He had no other medical history. Routine laboratory tests, urinalysis, stool examination and chest X-ray revealed no definite abnormality. Serology for HBV, HCV, HIV and a PPD test for TB were negative. As for case 1, antibody serology by ELISA on serum and CSF for schistosomiasis, echinococcosis, taeniasis solium, taeniasis bovis and toxoplasmosis was negative but positive for $S$. mansoni.

When interviewed, the patient's mother stated that the locals in her village often drank unboiled water from a nearby well, that there had been a recently confirmed cerebral sparganosis mansoni case in the village and surgery had been performed for removal of the parasite.
Axial MRI at the onset of symptoms (October, 2010) showed low signal on T1WI (Figure 2(A)) and high signal on T2WI (Figure 2(B)) images indicating edema at the central zone of the subcortex of the right parietal lobe. Lesions with the appearance of a twisted rope or a string of beads with ringlets (tunnel signal) changed location and shape from the onset of disease symptoms (Figure 2(C)), moving to the anterior cuneus at 6months follow-up (Figure 2(D)). An initial unenhanced axial CT (Figure 2(E)) showed a hypo-density edema, an isodensity nodule and pinpoint calcifications at the subcortex of the right parietal lobe. Enhanced axial CT (Figure 2(F)) showed the lesion manifested medium enhancement and heterogeneous density.

The patient had taken praziquantel once before admission to HIPD as prior diagnosis had suggested infection with a parasite. Other medication included oral carbamazepine tablets (100 mg twice daily from the onset of disease symptoms to hospital admission).

\section{DIAGNOSIS}

Both patients were diagnosed as having cerebral sparganosis and they were referred to other hospitals in Changsha (the capital city of Hunan province) with better facilities for brain surgery. To date, the parents of both patients are still considering whether to proceed with the physicians' recommendations of surgical removal of the parasites complemented with anti-epileptic therapy.

Although rare, cerebral sparganosis is far more severe than other forms of sparganosis [2-6]. Praziquantel drug treatment has minimal effect on human sparganosis so surgical removal of the parasite is the treatment of choice. The definitive diagnosis of cerebral sparganosis is usually made by histology after removal of the parasite by surgery [6]. For the two cases described here, pre-surgical diagnosis was, however, made based on the following criteria: 1) Hunan and Jiangxi provinces in China are known to be endemic for Spirometra mansoni [2,3]; a previous case of cerebral sparganosis mansoni was confirmed after surgery in the same village inhabited by case 2. 2) The occurrence of seizures and continuous headaches in both cases. 3) Evidence of eating under/uncooked frogs/snakes or drinking unboiled water. 4) CSF and serum serologically antibody-positive to $S$. mansoni antigen in ELISA. 5) Tunnel signal, appearing as a string of bead-shape/ring-like lesions or a twisted thread plus adjacent widening of local sulci, in enhanced MRI. Movement of the lesions over time showed that the parasite was still alive after praziquantel treatment. 6) Scattered multiple pinpoint calcifications evident in CT, due to the plerocercoid larva itself or substances (such as calcareous corpuscles) released from the body of the tapeworm [7]. 


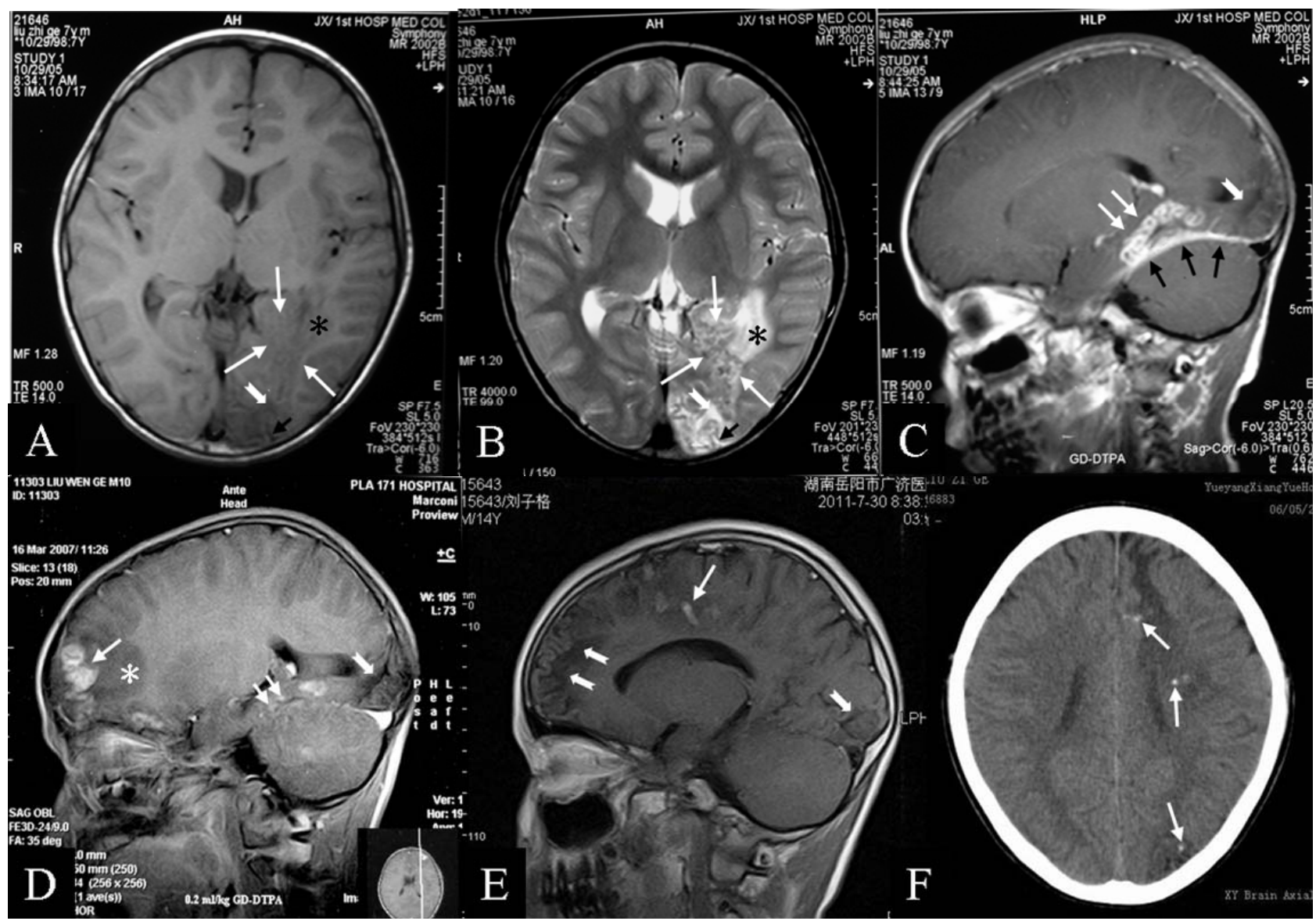

Figure 1. A 13-year-old male with a medical history of occasional seizures for 6 years (Case 1). An initial axial MRI at the onset of symptoms (October 29, 2005) shows a low signal lesion on T1-weighted spin-echo images (T1WI) (A) and a heterogeneous high signal on T2WI (B) in the left temporo-occipital lobe (white arrows). Edema (star) and widening of local sulci (short black arrows) are revealed on both T1WI and T2WI (A \& B). Initial sagittal enhanced MRI (C) shows a string of bead-shape or ring-like lesions (tunnel signal) (white arrows) in the left temporo-occipital lobe and abnormal enhancement of the adjacent dura mater (black arrows). After onset of symptoms, the lesion of a mass of threads (white arrows) then shifts from the frontal lobe at 16-months follow-up (D) and subsequently to the gyrus frontalis superior after 69-months (E). An encephalomalacia focus with the signal intensity similar to that of cerebrospinal fluid (open-end white arrow) is continually persistent at the left occipital pole from the initial signs of illness to the 69-month follow up (A-E). Another new encephalomalacia focus (open-end white arrow) is also found at the 69-month timepoint in the gyrus frontalis medius and inferior (E). Unenhanced CT undertaken in May 2011 revealed low-density areas both in left occipital and temporal lobes, and scattered pinpoint calcifications in the left cerebral hemisphere (white arrows) (F).

\section{DIFFERENTIATION}

The ring-like lesions in brain MRI or calcification in brain CT can also be found in diseases caused by other parasites such as cerebral schistosomiasis, cerebral paragonimiasis, cerebral echinococcosis, cerebral cysticercosis, cerebral toxoplasmosis, or in other disease syndromes such as cerebral tuberculoma or brain tumor. However, a definitive diagnosis for differentiating sparganosis from the other diseases in the two cases presented here was based on the fact that both were serologically negative for 5 other parasitic diseases but positive for sparganosis and the brain neuroimaging features were consistent with an infection of $S$. mansoni. Other contributory factors pointing to both cases being due to migrating cerebral sparganosis were as follows:
1) Cerebral schistosomiasis-Although Hunan and Jiangxi provinces are endemic for Asian schistosomiasis [8], the areas where both cases were resident are not endemic. Furthermore, neither subject had travelled to any schistosomiasis-endemic area. The nodular (silt-like) lesion caused by cerebral schistosomiasis can be observed in enhanced MRI instead of in CT [9].

2) Cerebral paragonimiasis-The migratory ring-like enhancing lesions in cerebral paragonimiasis are similar to those found in sparganosis $[4,5,10]$ but those in the former usually vary in size and are larger (sparganosis, less than $5 \mathrm{~mm}$ ), and their appearance is more grape-like.

The calcifications in cerebral paragonimiasis usually have a soap-bubble appearance and are clustered together $[4,10]$. No evidence of pulmonary symptoms or signs [10] 


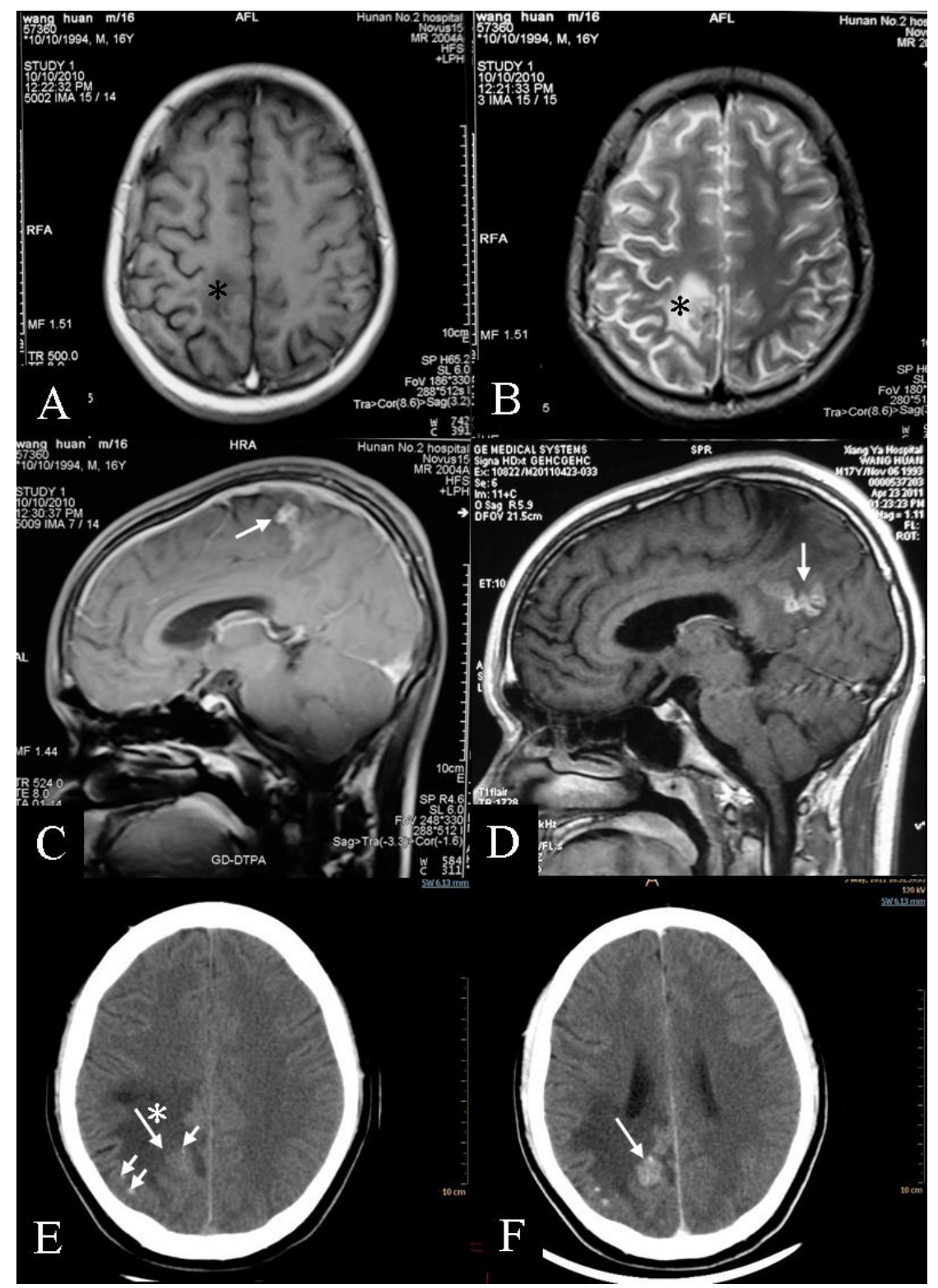

Figure 2. A 17-year-old male with occasional seizures for 9 months (Case 2). Initial axial MRI at onset of symptoms (October of 2010) shows low signal on T1WI (A) and high signal on T2WI (B) images indicating edema at the central zone of the subcortex of the right parietal lobe. Lesions with appearance of a twisted rope or a string of beads with ringlets (tunnel signal) change location and shape from the onset of disease symptoms (C) to the anterior cuneus at 6-months follow up (C). An initial unenhanced axial CT (E) shows a hypo-density edema (star), an isodensity nodule (long white arrow) and pinpoint calcifications (short white arrows) at the subcortex of the right parietal lobe. Enhanced axial CT (F) shows the lesion manifests with medium enhancement and heterogeneous density (long white arrow).

also ruled out this infection.

3) Cerebral echinococcosis-Ring-like lesions in cerebral echinococcosis are usually large, unilocular andthin-walled, with no calcification or surrounding oedema. They contain fluid with a similar density to CSF on CT and MRI. MRI may show particulate matter within the cyst due the presence of daughter protoscoleces and hy- datid sand. Most patients present with symptoms and signs of raised intracranial pressure including headache, nausea, vomiting and papilloedema [5,11]. Furthermore, echinococcosis is non-endemic to Hunan and Jiangxi provinces [3] and neither subject had travelled to north west China where the disease is common.

4) Cerebral cysticercosis-The images of cerebral cys- 
ticercotic lesions vary depending on the morphology of the larvae and the examination technique employed [4,5]. The individual ring-like enhancement of a lesion in MRI of cerebral cysticercosis is a characteristic "hole-withdot”, with the tapeworm scolex visible as a bright nodule within the cyst; round calcifications are larger and more even in size [12].

5) Cerebral toxoplasmosis-The protozoan parasite Toxoplasma gondii is the most common organism affecting the brain in AIDS patients. The lesions are slightly hypointense or isointense relative to the brain parenchyma on TIWI, and hyperintense or isointense on T2WI. Ring lesions commonly demonstrate non-enhancing central areas corresponding to coagulation necrosis. The rings are usually thin, smooth and may be nodular in shape, particularly in small lesions [13].

6) Cerebral tuberculoma-Cheesy narcosis is often revealed in the center of cerebral tuberculoma by MRI. Round, thick-walled, ring-like or nodular lesions can also be observed on MRI [14]. Hypointense lesions can be observed in T2WI and no migrating string of beads is observed in the cerebral parenchyma by imaging.

7) Brain tumor-Brain lesions caused by primary or metastatic tumors in CT/MRI can vary in shape and location but rarely migrate, nor do they appear as a string of beads [15].

\section{CONCLUSIONS}

MRI is the diagnostic modality of choice in the evaluation of most parasitic infections involving the CNS. It is superior to CT in detecting parasitic lesions, in characterizing the lesions, and in delineating the extent of parenchymal changes. Gadolinium-enhanced MRI is indispensable for both specific diagnosis of the disease and assessment of inflammatory activity, whereas CT is superior in identifying small calcifications [5]. In the two cases presented here, sparganosis was diagnosed based on neuroimaging characteristics but complementary epidemiological information and serology were also helpful.

Compared with other parasitic infections such as malaria [16] and schistosomiasis [8], human sparganosis is relatively rare, and is one of the most neglected parasitic diseases in China where the facilities for correct diagnosis are generally poor. In Hunan province, for example, there is only one medical facility (in Changsha) able to undertake serology for sparganosis. Furthermore, clinicians in general hospitals are not familiar with the CT and MRI features of sparganosis. Meanwhile, health education is needed to improve people's awareness of preventing infection by not drinking contaminated water and to make sure to cook food thoroughly. Although there have been a number of human sparganosis cases reported in the Chinese literature, epidemiological investigations are few [2]. Further surveillance of S. mansoni in mammalian hosts including cats, dogs and humans, and in reptiles and amphibians should be undertaken in Hunan province and other rural parts of China to determine the true extent of the problem of sparganosis.

\section{CONSENT}

Written informed consents were obtained from both patients' mother as next to kin for publication of these cases reports and any accompanying images.

\section{ACKNOWLEDGEMENTS}

We thank the patients' participation and their families' understanding to publish their information!

\section{REFERENCES}

[1] Mueller, J.F. (1974) The biology of spirometra. Journal of Parasitology, 60, 3-14. doi:10.2307/3278670

[2] Li, M.W. and Song, H.Q. (2011) Sparganosis in mainland China. International Journal of Infectious Diseases, 15, e154-e156. doi:10.1016/j.ijid.2010.10.001

[3] Zhan, X.M. (2005) Human parasitology. People’s Medical Publishing House, Beijing, 155-157. (in Chinese)

[4] Lv, S., Zhang, Y., Steinmann, P., Zhou, X.N. and Utzinger, J. (2010) Helminth infections of the central nervous system occurring in Southeast Asia and the Far East. Advances in Parasitology, 72, 351-408. doi:10.1016/S0065-308X(10)72012-1

[5] Chang, K.H. and Han, M.H. (1998) MRI of CNS parasitic diseases. Journal of Magnetic Resonance Imaging, 8, 297-307. doi:10.1002/jmri.1880080209

[6] Kim, D.G., Paek, S.H. and Chang, K.H. (1996) Cerebral sparganosis: Clinical manifestations, treatment, and outcome. Journal of Neurosurgery, 85, 1066-1071. doi:10.3171/jns.1996.85.6.1066

[7] Chiu, C.H., Chiou, T.L., Hsu, Y.H. and Yen, P.S. (2010) MR spectroscopy and MR perfusion character of cerebral sparganosis: A case report. British Journal of Radiology, 83, e31-e34. doi:10.1259/bjr/73038348

[8] Ross, A.G., Sleigh, A.C., Li, Y., Davis, G.M., Williams, G.M., Jiang, Z., Feng, Z. and McManus, D.P. (2001) Schistosomiasis in the People's Republic of China: Prospects and challenges for the 21st century. Clinical Microbiology Reviews, 14, 270-295. doi:10.1128/CMR.14.2.270-295.2001

[9] Li, Y., Ross, A.G., Hou, X., Lou, Z. and McManus, D.P. (2011) Oriental schistosomiasis with neurological complications: Case report. Annals of Clinical Microbiology and Antimicrobials, 7, 1-5.

[10] Im, J.G., Chang, K.H. and Reeder, M.M. (1997) Current diagnostic imaging of pulmonary and cerebral paragonimiasis, with pathological correlation. Seminars in Roentgenology, 32, 301-324. doi:10.1016/S0037-198X(97)80024-7

[11] Bükte, Y., Kemaloglu, S., Nazaroglu, H., Ozkan, U., Ce- 
viz, A. and Simsek, M. (2004) Cerebral hydatid disease: CT and MR imaging findings. Swiss Medical Weekly, 134, 459-467.

[12] Del Brutto, O.H., Rajshekhar, V. and White Jr., A.C. (2001) Proposed diagnostic criteria for neurocysticercosis. Neurology, 57, 177-183. doi:10.1212/WNL.57.2.177

[13] Ramsay, R.G. and Gerenia, G.K. (1988) CNS complications of AIDS: CT and MRI findings. American Journal of Roentgenology, 151, 449-454.

[14] Gupta, R.K., Pandey, P., Khan, E.M., Mittal, P., Gujral, R.B. and Chhabra, D.K. (1993) Intracranial tuberculomas:
MRI signal intensity correlation with histopathology and localized proton spectroscopy. Magnetic Resonance Imaging, 11, 443-449. doi:10.1016/0730-725X(93)90079-S

[15] Ricard, D., Idbaih, A., Ducray, F., Lahutte, M., HoangXuan, K. and Delattre, J.Y. (2012) Primary brain tumours in adults. Lancet, 379, 1984-1996. doi:10.1016/S0140-6736(11)61346-9

[16] Yang, G.J., Gao, Q. and Zhou, S.S. (2010) Mapping and predicting malaria transmission in the People's Republic of China, using integrated biology-driven and statistical models. Geospatial Health, 5, 11-22. 